\title{
Pre-Plant Soaking of Corms in Growth Regulators Influences the Multiple Sprouting, Floral and Corm Associated Traits in Gladiolus grandiflorus L.
}

\author{
Yasar Sajjad ${ }^{1,2}$, Muhammad J. Jaskani ${ }^{2}$, Muhammad Qasim², Asim Mehmood ${ }^{3}$, Naseer Ahmad $^{1} \&$ Gulzar Akhtar $^{4}$ \\ ${ }^{1}$ Department of Environmental Sciences, COMSATS Institute of Information Technology, Abbottabad, Pakistan \\ ${ }^{2}$ Institute of Horticultural Sciences, University of Agriculture, Faisalabad, Pakistan \\ ${ }^{3}$ Department of Biosciences, COMSATS Institute of Information Technology, Sahiwal, Pakistan \\ ${ }^{4}$ Department of Horticulture, College of Agriculture, University of Sargodha, Pakistan \\ Correspondence: Yasar Sajjad, Department of Environmental Sciences, COMSATS Institute of Information \\ Technology, Abbottabad, Pakistan. E-mail: yasarsajjad@ciit.net.pk
}

Received: May 20, 2015 Accepted: July 13, 2015 Online Published: August 15, 2015

doi:10.5539/jas.v7n9p173 URL: http://dx.doi.org/10.5539/jas.v7n9p173

\begin{abstract}
Gladiolus is an ornamental bulbous plant and is commercially propagated in the world through its corms. Usually, a single bud is sprouted from a mother corm but sprouting of multiple buds favors an increase in production of propagules. Thus, present research work was carried out to enhance the sprouting of multiple buds and evaluate its effects on other growth parameters through pre-plant soaking of corms in solution of plant growth regulators. The corms were soaked in solutions of gibberellic acid $\left(\mathrm{GA}_{3}\right)$, benzyladenine $(\mathrm{BA})$ or ethrel at 0, 50, 100 or $150 \mathrm{ppm}$ concentrations for $24 \mathrm{hrs}$ before planting in the field. Gibberellic acid at 100ppm concentration increased plant height to $105 \mathrm{~cm}$ compared to $97.60 \mathrm{~cm}$ in control plants, flowering percentage $(84.67 \%)$, spike length $(40.03 \mathrm{~cm})$ and also boosted the corm weight $(68.30 \mathrm{~g})$. Soaking of corms in $150 \mathrm{ppm}$ benzyladenine solution enhanced the number of sprouting per corm (2.14) and reduced the plant height $(87.00$ $\mathrm{cm})$ while $50 \mathrm{ppm}$ concentration of ethrel increased the spike length $(42.14 \mathrm{~cm})$. In conclusion, soaking of corms in benzyladenine favored the modifications in various traits of interest including sprouting of multiple buds and an increase in the production of corms while gibberellic acid improved the floral characteristics of gladiolus spikes.
\end{abstract}

Keywords: corm treatment, corm multiplication, gladiolus, plant growth regulators, production system

\section{Introduction}

Gladiolus is a perennial bulbous plant that belongs to family Irridaceae and widely grown as a cut flower in the world. The genus "Gladiolus" consists of 260 species, out of which 250 belong to sub-Saharan Africa while 10 species from Eurasia (Goldblatt \& Manning, 1998; Manning \& Goldblatt, 2008). The gladiolus is commercially propagated through its corm which is an underground modified stem that provides nutrients during sprouting (Ghamsari et al., 2007).

The conventional production system of gladiolus allows to get usually one sprout and one daughter corm from a mother corm but sprouting of more buds from a single corm favors to increase in production of corms. The minimum control over vegetative and floral traits is another drawback of conventional system, which is not in favor of meeting the market demands. The control over flowering time and floral characteristics according to the demand of market has been achieved in many cut flowers by adopting modern production techniques including the use of plant growth regulators (PGRs). The application of PGRs has become the part of their cultural practices in many ornamental plants to modify their vegetative and floral traits. There are different application methods of PGRs including foliar application, pre-plant soaking and drenching but foliar application is the most common method being practiced in ornamental plants. Soaking of bulbs in solution of plant growth regulators has been used (Larson et al., 1987) and it is an efficient method for obtaining good results and also have advantages over other methods in terms of time, labor saving and accurate dosage (Ranwala et al., 2002). Keeping in view the efficiency of this method, Pre-plant dipping of planting material in a chemical solution is 
now becoming a popular method among commercial growers (Schnelle et al., 2005).

PGRs consist of numerous compounds including gibberellins, auxins, cytokinins, abscisic acid and ethylene. The use of plant growth regulators is a successful approach for improvement of various traits, including, number of flower buds (Boyle, 1992), induction of early flowering (Sakai et al., 1979), delay flowering (Paulin \& Muloway, 1979), flower development (Irish, 2009) and increase yield (Malabug et al., 2010). PGRs has been used in the commercial production of ornamental plants (Rademacher, 1991) but their efficacy depends on different plant species (Dole \& Wilkins, 1999).

Gibberellic acid has an important role in different plant processes, including seed germination, stem elongation, leaf expansion and flower development (Olszewski et al., 2002). Ethrel is a chemical compound containing ethephon as an active ingredient, releases ethylene which act as plant growth regulators. Application of ethrel is effective in modifying different attributes of ornamental plants. The cytokinins are an important part of several commercial plant growth regulators (PGRs) being used on different plant. Application of Cytokinins has been reported to stimulate the growth of axillary buds in plants. Benzyladenine is also a cytokinin and its application is reported in different ornamental bulbous plants including tulips (de Munk \& Gijzenberg, 1977), lilies (Ranwala \& Miller, 1998) and calla bulbs (Naor et al., 2005).

The objective of this study was to evaluate the effects of pre-plant application of PGRs on sprouting of multiple buds, production of corms, cormels, floral traits, and also to find out interaction among these traits, that can help to improve the conventional production system of gladiolus.

\section{Materials and Methods}

\subsection{Plant Material and Treatments}

The healthy corms of gladiolus cultivar "Amsterdam" were purchased from the local market of Lahore, Pakistan. The corms were dipped in a freshly prepared solution of gibberellic acid, benzyladenine or ethrel at different concentrations of 50,100 or $150 \mathrm{ppm}$ for $24 \mathrm{hrs}$. The control corms were dipped in distilled water for the same time. The treated and non treated corms were planted in the field of floriculture research area, University of Agriculture, Faisalabad, Pakistan, on ridges at the plant to plant distance of $15 \mathrm{~cm}$ while row to row distance was $60 \mathrm{~cm}$. The field area is located at $31^{\circ} 26^{\prime} \mathrm{N}$ latitude, $73^{\circ} 06^{\prime} \mathrm{E}$ longitude and $184.4 \mathrm{~m}$ altitude. All the cultural practice including irrigation, weeding, fertilizers were same for all plants. The irrigation was scheduled at the interval of 7-10 days according to the condition of soil while NPK fertilizer was applied at 100, 90, $100 \mathrm{~kg} / \mathrm{acre}$, respectively and weeding was done manually. The experiment was conducted in randomized complete block design (RCBD) with three replicates having 60 corms in each treatment.

\subsection{Data Collection}

The plants were allowed to grow in the field and data was recorded according to the growth stage of plants. The data for different vegetative variables, including days to sprouting, number of buds sprouted per corm, vegetative plant height $(\mathrm{cm})$ was recorded before the initiation of flowering while data for total plant height $(\mathrm{cm})$, days to open $1^{\text {st }}$ floret, spike length $(\mathrm{cm})$, spike diameter $(\mathrm{cm}), 1^{\text {st }}$ floret weight $(\mathrm{g}), 1^{\text {st }}$ floret diameter $(\mathrm{cm})$ and flowering percentage was noted after the opening of florets in the spike. Lifting of corms from the field was done after 70 days of flowering, and the data for corm related attributes including, corm diameter $(\mathrm{cm})$, corm weight $(\mathrm{g})$, number of corms per plant, total number of cormels per plant, total cormel weight per plant ( $\mathrm{g}$ ) and average weight of cormel $(\mathrm{g})$ was recorded.

\subsection{Statistical Analysis}

The recorded data was subjected to analysis of variance and treatment means were compared by applying Duncan'S Multiple Range (DMR) test by using SAAS software version 1.101. Pearson correlation among different variables was found by using SPSS ver. 16 software.

\section{Results}

\subsection{Vegetative Variables}

Corms treated with gibberellic acid showed $100 \%$ sprouting within 10 days of sowing compared to $75 \%$ sprouting in control (Figure 1). Benzyladenine delayed the sprouting process and $56 \%$ sprouting recorded in 10 days. All the treatment showed $100 \%$ sprouting but after 15 days of sowing. The significant effect of treatments on the number of sprouted buds per corm was shown in Table 1 . The control corms showed only 1.02 sprouts per corm while benzyladenine treatment at $150 \mathrm{ppm}$ increased the sprouting of buds to 2.14 followed by 1.69 at 100 $\mathrm{ppm}$. Foliage plant height also increased by the application of $100 \mathrm{ppm}$ ethrel to $78.26 \mathrm{~cm}$ compared to $70.18 \mathrm{~cm}$ in control plants. Gibberellic acid at $100 \mathrm{ppm}$ concentration also increased the foliage plant height $(75.26 \mathrm{~cm})$. 
The soaking of corms in gibberellic acid at $100 \mathrm{ppm}$ concentration significantly increased the total height of gladiolus plants to $105.80 \mathrm{~cm}$ compared to $97.60 \mathrm{~cm}$ in control plants. The maximum plant height $(110.98 \mathrm{~cm})$ was recorded in the $100 \mathrm{ppm}$ concentration of ethrel treatment.

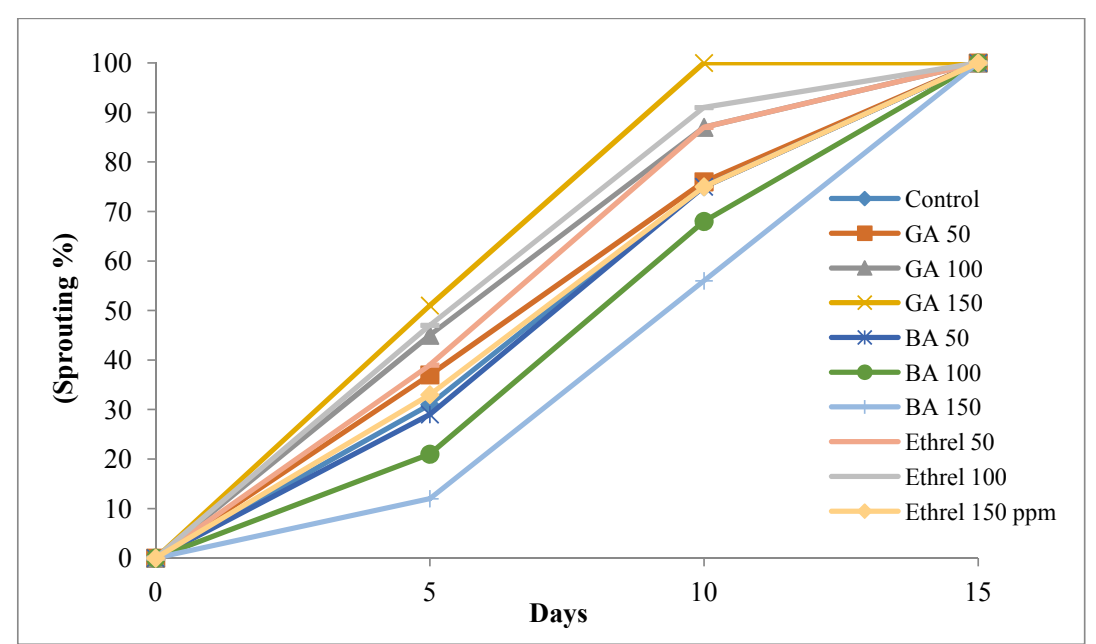

Figure 1. Effect of growth regulators on sprouting percentage of gladiolus corms

\subsection{Floral Variables}

Application of plant growth regulators also changed the flower characteristics of gladiolus spikes. Gibberellic acid at $100 \mathrm{ppm}$ concentration significantly decreased the days (70.88) to opening of $1^{\text {st }}$ floret on the spike followed by 70.97 days in $50 \mathrm{ppm}$ of ethrel (Table 2). Benzyladenine caused the significant delay in flowering and its $150 \mathrm{ppm}$ application increased days to opening of $1^{\text {st }}$ floret to 107.50 days compared to 77.05 days in control plants. Flowering percentage increased to $84.67 \%$ in the treatment of $100 \mathrm{ppm}$ gibberellic acid compared to $71.67 \%$ in control plants. Benzyladenine also increased the flowering percentage $(80.33 \%)$ in $50 \mathrm{ppm}$ treatment, but decreased in comparatively higher concentrations and minimum flowering percentage $(21 \%)$ was recorded in the $150 \mathrm{ppm}$ dose.

Table 1. Effect of growth regulators on vegetative variables of gladiolus

\begin{tabular}{lllll}
\hline Treatments $(\mathrm{ppm})$ & & Sprouted buds per corm & Foliage Plant Height $(\mathrm{cm})$ & Total Plant Height $(\mathrm{cm})$ \\
\hline Control & & $1.02 \pm 0.02 \mathrm{e}$ & $70.18 \pm 0.76 \mathrm{~d}$ & $97.60 \pm 1.50 \mathrm{~cd}$ \\
\hline \multirow{2}{*}{$\mathrm{GA}_{3}$} & 50 & $1.06 \pm 0.04 \mathrm{de}$ & $71.06 \pm 0.04 \mathrm{~d}$ & $99.63 \pm 1.95 \mathrm{c}$ \\
& 100 & $1.29 \pm 0.08 \mathrm{cde}$ & $75.26 \pm 1.67 \mathrm{bc}$ & $105.80 \pm 0.35 \mathrm{~b}$ \\
& 150 & $1.25 \pm 0.14 \mathrm{cde}$ & $74.58 \pm 1.49 \mathrm{bc}$ & $105.47 \pm 0.65 \mathrm{~b}$ \\
\hline \multirow{3}{*}{ BA } & 50 & $1.44 \pm 0.04 \mathrm{bc}$ & $72.38 \pm 0.22 \mathrm{~cd}$ & $100.79 \pm 0.70 \mathrm{c}$ \\
& 100 & $1.69 \pm 0.04 \mathrm{~b}$ & $70.00 \pm 1.48 \mathrm{~d}$ & $93.13 \pm 2.24 \mathrm{e}$ \\
& 150 & $2.14 \pm 0.08 \mathrm{a}$ & $64.20 \pm 0.55 \mathrm{e}$ & $87.00 \pm 0.58 \mathrm{f}$ \\
\hline \multirow{2}{*}{ Ethrel } & 50 & $1.13 \pm 0.07 \mathrm{de}$ & $76.90 \pm 2.01 \mathrm{ab}$ & $110.98 \pm 2.15 \mathrm{a}$ \\
& 100 & $1.32 \pm 0.22 \mathrm{~cd}$ & $78.26 \pm 0.84 \mathrm{a}$ & $109.20 \pm 0.35 \mathrm{ab}$ \\
& 150 & $1.01 \pm 0.01 \mathrm{e}$ & $70.90 \pm 1.67 \mathrm{~d}$ & $94.25 \pm 0.72 \mathrm{de}$
\end{tabular}

Note. $\mathrm{GA}_{3}$ : Gibberellic acid; BA: Benzyladenine. Means sharing the same letter do not differ by DMR test at $5 \%$ probability level; \pm means SE.

Control plants showed spike length of $34.75 \mathrm{~cm}$ and maximum increase $(42.14 \mathrm{~cm})$ was recorded in $50 \mathrm{ppm}$ treatment of ethrel. Spike diameter was also increased to $0.86 \mathrm{~cm}$ in $50 \mathrm{ppm}$ ethrel compared to $0.75 \mathrm{~cm}$ in control plants. Application of $100 \mathrm{ppm}$ ethrel showed a maximum increase (12.88) in a number of florets per spike while the minimum increase (9.90) was recorded in $150 \mathrm{ppm}$ ethrel. The maximum increase in floret 
diameter $(8.98 \mathrm{~cm})$ was exhibited in the $150 \mathrm{ppm}$ concentration of gibberellic acid while benzyladenine at same concentration showed a minimum increase $(6.31 \mathrm{~cm})$ in floret diameter. The $1^{\text {st }}$ floret weight was increased to $5.27 \mathrm{~g}$ in application of $150 \mathrm{ppm}$ gibberellic acid compared to $4.27 \mathrm{~g}$ in control plants.

\subsection{Corms Variables}

The treatments significantly affected the different attributes of corms and cormels (Table 3). Benzyladenine at $150 \mathrm{ppm}$ concentration showed a maximum increase (2.50) in the number of corms per plant while the minimum increase in corm diameter $(3.90 \mathrm{~cm})$ was also recorded in this treatment. Benzyladenine induced the multiple shoot induction which also resulted in production of multiple daughter corms from a single parent corm as shown in figure 2. Application of gibberellic acid at $100 \mathrm{ppm}$ caused the maximum increase in corm diameter $(6.01 \mathrm{~cm})$ compared to $5.81 \mathrm{~cm}$ in control plants. Corm weight was significantly increased $(68.30 \mathrm{~g})$ in response to application of $100 \mathrm{ppm}$ gibberellic acid followed by $66.51 \mathrm{~g}$ in the $100 \mathrm{ppm}$ concentration of ethrel. The highest increase in the number of cormels per plant (31.33) was observed in gibberellic acid treatment at 150 ppm concentration while 8.57 cormels per plant recorded in control plants. Total cormel weight per plant was increased to $5.96 \mathrm{~g}$ in $150 \mathrm{ppm}$ concentration of ethrel while control plants gave the maximum increase in average cormel weight $(0.41 \mathrm{~g})$.

\subsection{Correlation among Variables}

Number of sprouted buds per corm showed significant negative correlation with the other vegetative, floral and corm related parameters except the corms per plant (Table 4). Sprouting of buds per corm is significantly affected by the application of PGRs especially the benzyladenine. This parameter greatly influenced on altering the other valuable parameters and found as key to control the plant growth in the present research. The second most influential parameter was days to flower which showed negative correlation with the floral and corm associated parameters except the corms per plant which is positively correlated. On the other hand, vegetative plant height showed significantly positive correlation with all parameters of floral and corm growth except days to flower and corms per plant which are negatively correlated. Parameters associated with cormels are not significantly correlated with the vegetative and floral variables, but the number of cormel per plant showed significant correlation with other parameters of cormels including total cormel weight and average cormel weight. It means that the total weight of cormels increased with the increase in the total number of cormels but the average weight of a cormel decreased.

Table 2. Effects of growth regulators on floral variables of gladiolus

\begin{tabular}{|c|c|c|c|c|c|c|c|c|}
\hline \multicolumn{2}{|c|}{$\begin{array}{l}\text { Treatments } \\
(\mathrm{ppm})\end{array}$} & Days to flower & Flowering $\%$ & $\begin{array}{l}\text { Spike length } \\
(\mathrm{cm})\end{array}$ & $\begin{array}{l}\text { Spike diameter } \\
(\mathrm{cm})\end{array}$ & Florets per spike & $\begin{array}{l}\text { Floret diameter } \\
(\mathrm{cm})\end{array}$ & $\begin{array}{l}\text { Floret weight } \\
\text { (g) }\end{array}$ \\
\hline \multicolumn{2}{|l|}{ Control } & $77.05 \pm 1.10$ cde & $71.67 \pm 3.53 \mathrm{~d}$ & $34.57 \pm 0.82 \mathrm{~cd}$ & $0.75 \pm 0.01 \mathrm{~cd}$ & $10.24 \pm 0.05 \mathrm{de}$ & $7.95 \pm 0.19 \mathrm{c}$ & $4.27 \pm 0.45 \mathrm{~cd}$ \\
\hline \multirow{3}{*}{$\mathrm{GA}_{3}$} & 50 & $73.80 \pm 1.15 \mathrm{def}$ & $72.67 \pm 2.33 \mathrm{~d}$ & $37.85 \pm 0.95 \mathrm{bc}$ & $0.78 \pm 0.01 \mathrm{~b}$ & $11.25 \pm 0.14 \mathrm{bc}$ & $8.27 \pm 0.13 b c$ & $4.31 \pm 0.49 \mathrm{c}$ \\
\hline & 100 & $70.88 \pm 0.65 \mathrm{f}$ & $84.67 \pm 2.40 \mathrm{a}$ & $40.03 \pm 0.79 \mathrm{ab}$ & $0.79 \pm 0.02 \mathrm{~b}$ & $12.78 \pm 0.01 \mathrm{a}$ & $8.94 \pm 0.41 \mathrm{a}$ & $5.23 \pm 0.42 \mathrm{a}$ \\
\hline & 150 & $71.85 \pm 0.20$ ef & $83.33 \pm 2.73 \mathrm{ab}$ & $37.69 \pm 0.01 b c$ & $0.76 \pm 0.04 \mathrm{bc}$ & $11.83 \pm 0.25 b$ & $8.98 \pm 0.20 \mathrm{a}$ & $5.27 \pm 0.13 \mathrm{a}$ \\
\hline \multirow{3}{*}{ BA } & 50 & $82.25 \pm 1.59 \mathrm{c}$ & $82.33 \pm 2.19 \mathrm{ab}$ & $36.44 \pm 0.74 \mathrm{bcd}$ & $0.72 \pm 0.01 \mathrm{de}$ & $10.68 \pm 0.04 \mathrm{~cd}$ & $8.23 \pm 0.28 b c$ & $4.97 \pm 0.24 \mathrm{ab}$ \\
\hline & 100 & $90.71 \pm 1.71 \mathrm{~b}$ & $46.00 \pm 2.65 \mathrm{f}$ & $33.17 \pm 1.06 \mathrm{~d}$ & $0.72 \pm 0.04 \mathrm{de}$ & $10.17 \pm 0.10 \mathrm{de}$ & $6.73 \pm 0.07 \mathrm{~d}$ & $4.26 \pm 0.03 \mathrm{~cd}$ \\
\hline & 150 & $107.50 \pm 4.91 \mathrm{a}$ & $21.00 \pm 2.08 \mathrm{~g}$ & $27.50 \pm 0.87 \mathrm{e}$ & $0.67 \pm 0.03 \mathrm{f}$ & $8.50 \pm 0.29 \mathrm{f}$ & $6.31 \pm 0.17 \mathrm{~d}$ & $3.80 \pm 0.33 \mathrm{~d}$ \\
\hline \multirow{3}{*}{ Ethrel } & 50 & $70.97 \pm 0.21 \mathrm{f}$ & $78.67 \pm 2.03 \mathrm{bc}$ & $42.14 \pm 0.08 \mathrm{a}$ & $0.86 \pm 0.05 \mathrm{a}$ & $12.83 \pm 0.10 \mathrm{a}$ & $8.64 \pm 0.04 \mathrm{ab}$ & $4.67 \pm 0.20 b c$ \\
\hline & 100 & $78.63 \pm 3.25 \mathrm{~cd}$ & $75.67 \pm 1.86 \mathrm{~cd}$ & $38.70 \pm 3.52 \mathrm{abc}$ & $0.80 \pm 0.01 \mathrm{~b}$ & $12.88 \pm 0.65 \mathrm{a}$ & $8.66 \pm 0.16 \mathrm{ab}$ & $4.66 \pm 0.22 b c$ \\
\hline & 150 & $79.20 \pm 1.85 \mathrm{~cd}$ & $59.67 \pm 2.91 \mathrm{e}$ & $37.75 \pm 0.72 b c$ & $0.71 \pm 0.03$ ef & $9.90 \pm 0.06 \mathrm{e}$ & $8.22 \pm 0.02 b c$ & $4.51 \pm 0.48 \mathrm{bc}$ \\
\hline
\end{tabular}

Note. BA: Benzyladenine; $\mathrm{GA}_{3}$ : Gibberellic acid; Means sharing the same letter do not differ by DMR test at $5 \%$ probability level; \pm means SE. 
Table 3. Effects of growth regulators on corm and cormels associated variables of gladiolus

\begin{tabular}{|c|c|c|c|c|c|c|c|}
\hline $\begin{array}{l}\text { Treatments } \\
(\mathrm{ppm})\end{array}$ & & Corms per plant & $\begin{array}{l}\text { Corm diameter } \\
(\mathrm{cm})\end{array}$ & $\begin{array}{l}\text { Corm weight } \\
\text { (g) }\end{array}$ & Comels per plant & $\begin{array}{l}\text { Total cormel wt } \\
(\mathrm{g})\end{array}$ & $\begin{array}{l}\text { Av. Cormel wt } \\
\text { (g) }\end{array}$ \\
\hline Control & & $1.01 \pm 0.01 \mathrm{~d}$ & $5.81 \pm 0.20 \mathrm{abc}$ & $57.70 \pm 4.88 \mathrm{bc}$ & $8.57 \pm 1.02 \mathrm{e}$ & $3.08 \pm 0.75 \mathrm{c}$ & $0.41 \pm 0.072 \mathrm{a}$ \\
\hline \multirow{3}{*}{$\mathrm{GA}_{3}$} & 50 & $1.21 \pm 0.04 \mathrm{~cd}$ & $5.90 \pm 0.05 \mathrm{ab}$ & $59.47 \pm 3.13 \mathrm{abc}$ & $18.17 \pm 0.29 \mathrm{~cd}$ & $3.90 \pm 0.08 b c$ & $0.28 \pm 0.071 b$ \\
\hline & 100 & $1.40 \pm 0.16 \mathrm{c}$ & $6.01 \pm 0.05 \mathrm{a}$ & $68.30 \pm 2.41 \mathrm{a}$ & $23.48 \pm 2.20 \mathrm{~b}$ & $4.58 \pm 0.90 \mathrm{~b}$ & $0.24 \pm 0.032 \mathrm{bcd}$ \\
\hline & 150 & $1.25 \pm 0.14 \mathrm{~cd}$ & $5.59 \pm 0.19 \mathrm{bcd}$ & $65.93 \pm 4.77 \mathrm{ab}$ & $31.33 \pm 3.95 \mathrm{a}$ & $3.77 \pm 0.53 b c$ & $0.13 \pm 0.02 \mathrm{~d}$ \\
\hline \multirow{3}{*}{ BA } & 50 & $1.19 \pm 0.11 \mathrm{~cd}$ & $5.46 \pm 0.20 \mathrm{~cd}$ & $54.70 \pm 3.25 \mathrm{c}$ & $17.43 \pm 0.74 \mathrm{~d}$ & $3.42 \pm 0.21 b c$ & $0.17 \pm 0.01 \mathrm{bcd}$ \\
\hline & 100 & $1.86 \pm 0.25 b$ & $4.40 \pm 0.16 \mathrm{e}$ & $37.11 \pm 4.92 \mathrm{~d}$ & $22.69 \pm 0.97 b c$ & $4.52 \pm 0.54 \mathrm{~b}$ & $0.26 \pm 0.06 \mathrm{~b}$ \\
\hline & 150 & $2.50 \pm 0.21 \mathrm{a}$ & $3.90 \pm 0.06 \mathrm{f}$ & $26.79 \pm 0.82 \mathrm{e}$ & $19.36 \pm 1.86 \mathrm{bcd}$ & $2.84 \pm 0.17 \mathrm{c}$ & $0.20 \pm 0.02 \mathrm{bcd}$ \\
\hline \multirow{3}{*}{ Ethrel } & 50 & $1.15 \pm 0.03 \mathrm{~cd}$ & $5.37 \pm 0.16 \mathrm{~d}$ & $51.48 \pm 2.44 \mathrm{c}$ & $19.25 \pm 1.20 \mathrm{bcd}$ & $3.67 \pm 0.01 \mathrm{bc}$ & $0.14 \pm 0.05 \mathrm{~cd}$ \\
\hline & 100 & $1.14 \pm 0.01 \mathrm{~cd}$ & $5.69 \pm 0.22 \mathrm{abcd}$ & $66.51 \pm 1.51 \mathrm{ab}$ & $30.59 \pm 0.01 \mathrm{a}$ & $4.17 \pm 0.09 b c$ & $0.23 \pm 0.07 \mathrm{bcd}$ \\
\hline & 150 & $1.08 \pm 0.05 \mathrm{~d}$ & $5.91 \pm 0.09 \mathrm{ab}$ & $60.10 \pm 4.73 \mathrm{abc}$ & $28.79 \pm 0.84 \mathrm{a}$ & $5.96 \pm 0.06 \mathrm{a}$ & $0.24 \pm 0.06 \mathrm{bc}$ \\
\hline
\end{tabular}

Note. BA: Benzyladenine; $\mathrm{GA}_{3}$ : Gibberellic acid; Means sharing the same letter do not differ by DMR test at $5 \%$ probability level; \pm means SE.

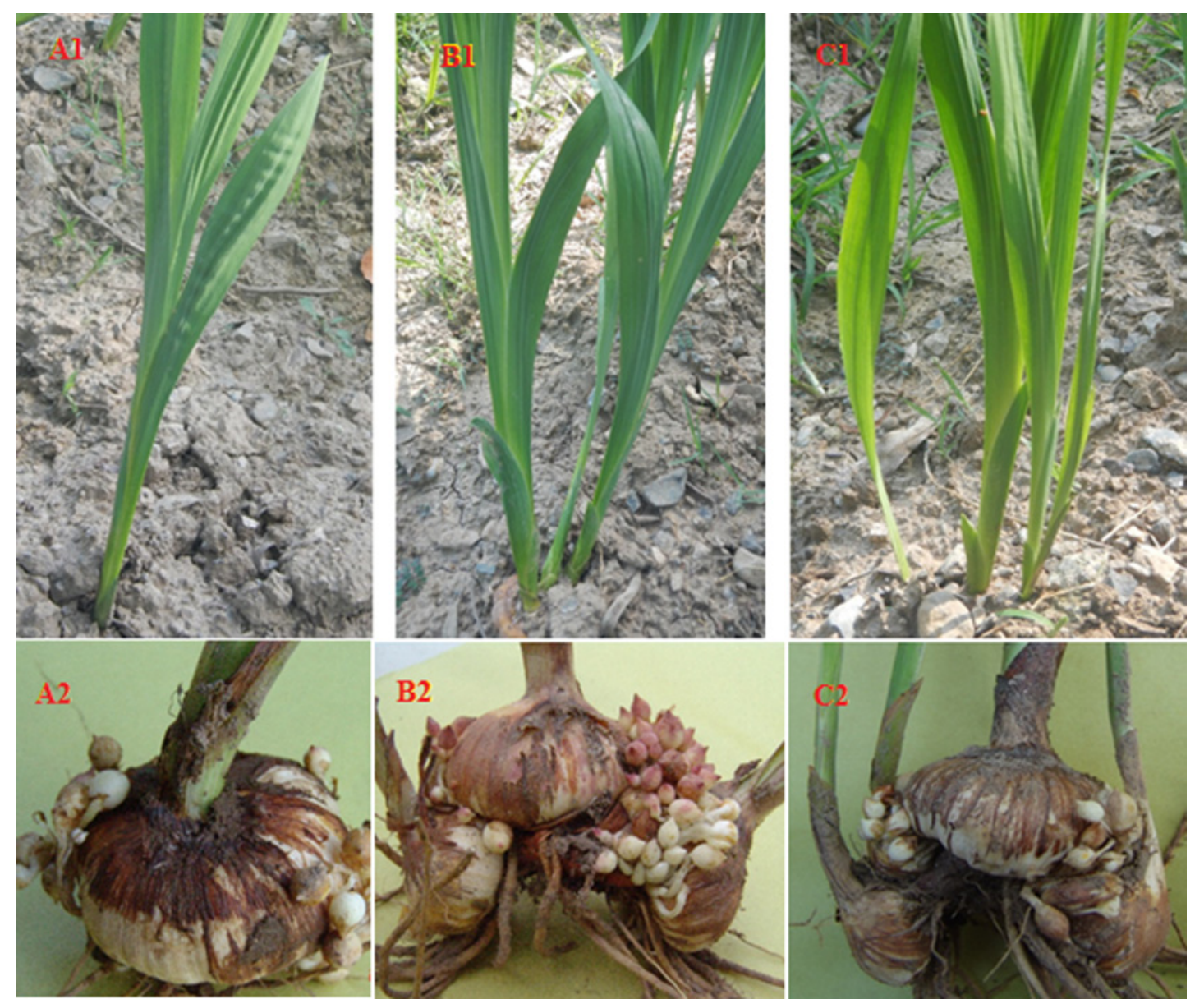

Figure 2. Effect of growth regulators on multiple shoot induction and corm multiplication in gladiolus, shoot induction (A1. Control., B1,C1. 150 ppm BA treated plants), corm multiplication (A2. Control., B2,C2. 150 ppm BA treated plants) 
Table 4. Pearson correlation coefficients among vegetative, floral and corm associated variables

\begin{tabular}{|c|c|c|c|c|c|c|c|c|c|c|c|c|c|c|c|}
\hline Variables & 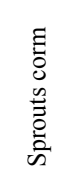 & 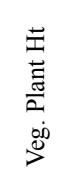 & 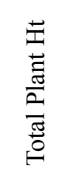 & 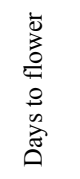 & $\begin{array}{l}a \\
\frac{d}{d} \\
\frac{0}{0} \\
\frac{0}{1}\end{array}$ & 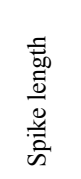 & 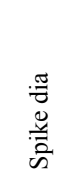 & 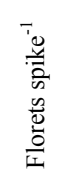 & 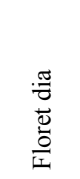 & $\begin{array}{l}\frac{5}{3} \\
\frac{0}{0} \\
\frac{0}{1} \\
\text { I. }\end{array}$ & 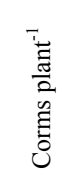 & 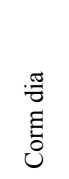 & $\begin{array}{l}3 \\
\vdots \\
0\end{array}$ & 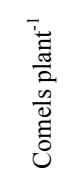 & 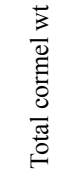 \\
\hline $\begin{array}{l}\text { Veg. Plant } \\
\mathrm{Ht}\end{array}$ & $-.426^{*}$ & & & & & & & & & & & & & & \\
\hline Total Plant & -.500 & .857 & & & & & & & & & & & & & \\
\hline $\mathrm{Ht}$ & $* *$ & $* *$ & & & & & & & & & & & & & \\
\hline Days to & .829 & -.658 & -.727 & & & & & & & & & & & & \\
\hline flower & $* *$ & $* *$ & $* *$ & & & & & & & & & & & & \\
\hline Flowering & -.685 & .741 & .787 & -.844 & & & & & & & & & & & \\
\hline$\%$ & $* *$ & $* *$ & $* *$ & $* *$ & & & & & & & & & & & \\
\hline Spike length & $\begin{array}{l}-.744 \\
* *\end{array}$ & $\begin{array}{l}.696 \\
* *\end{array}$ & $\begin{array}{l}.800 \\
* *\end{array}$ & $\begin{array}{l}-.838 \\
* *\end{array}$ & $\begin{array}{l}.763 \\
* *\end{array}$ & & & & & & & & & & \\
\hline $\begin{array}{l}\text { Spike } \\
\text { diameter }\end{array}$ & $\begin{array}{l}-.459 \\
*\end{array}$ & $\begin{array}{l}.732 \\
* *\end{array}$ & $\begin{array}{l}.873 \\
* *\end{array}$ & $\begin{array}{l}-.729 \\
* *\end{array}$ & $\begin{array}{l}.661 \\
* *\end{array}$ & $\begin{array}{l}.769 \\
* *\end{array}$ & & & & & & & & & \\
\hline $\begin{array}{l}\text { Florets per } \\
\text { spike }\end{array}$ & $\begin{array}{l}-.498 \\
* *\end{array}$ & $\begin{array}{l}.818 \\
* *\end{array}$ & $\begin{array}{l}.907 \\
* *\end{array}$ & $\begin{array}{l}-.731 \\
* *\end{array}$ & $\begin{array}{l}.739 \\
* *\end{array}$ & $\begin{array}{l}.847 \\
* *\end{array}$ & $\begin{array}{l}.868 \\
* *\end{array}$ & & & & & & & & \\
\hline $\begin{array}{l}\text { Floret } \\
\text { diameter }\end{array}$ & $\begin{array}{l}-.731 \\
* *\end{array}$ & $\begin{array}{l}.672 \\
* *\end{array}$ & $\begin{array}{l}.791 \\
* *\end{array}$ & $\begin{array}{l}-.860 \\
* *\end{array}$ & $\begin{array}{l}.821 \\
* *\end{array}$ & $\begin{array}{l}.781 \\
* *\end{array}$ & $\begin{array}{l}.622 \\
* *\end{array}$ & $\begin{array}{l}.769 \\
* *\end{array}$ & & & & & & & \\
\hline Floret weight & $-.409^{*}$ & $\begin{array}{l}.430 \\
*\end{array}$ & $\begin{array}{l}.452 \\
*\end{array}$ & $\begin{array}{l}-.504 \\
* *\end{array}$ & $\begin{array}{l}.466 \\
* *\end{array}$ & $\begin{array}{l}.519 \\
* *\end{array}$ & $\begin{array}{l}.377 \\
*\end{array}$ & $\begin{array}{l}.539 \\
* *\end{array}$ & $\begin{array}{l}.579 \\
* *\end{array}$ & & & & & & \\
\hline $\begin{array}{l}\text { Corms per } \\
\text { plant }\end{array}$ & $\begin{array}{l}.819 \\
* *\end{array}$ & $\begin{array}{l}-.493 \\
* *\end{array}$ & $\begin{array}{l}-.618 \\
* *\end{array}$ & $\begin{array}{l}.849 \\
* *\end{array}$ & $\begin{array}{l}-.776 \\
* *\end{array}$ & $\begin{array}{l}-.667 \\
* *\end{array}$ & $\begin{array}{l}-.521 \\
* *\end{array}$ & $\begin{array}{l}-.497 \\
* *\end{array}$ & $\begin{array}{l}-.784 \\
* *\end{array}$ & $\begin{array}{l}-.543 \\
* *\end{array}$ & & & & & \\
\hline $\begin{array}{l}\text { Corm } \\
\text { diameter }\end{array}$ & $\begin{array}{l}-.791 \\
* *\end{array}$ & $\begin{array}{l}.486 \\
* *\end{array}$ & $\begin{array}{l}.549 \\
* *\end{array}$ & $\begin{array}{l}-.821 \\
* *\end{array}$ & $\begin{array}{l}.790 \\
* *\end{array}$ & $\begin{array}{l}.625 \\
* *\end{array}$ & $\begin{array}{l}.438 \\
*\end{array}$ & $\begin{array}{l}.526 \\
* *\end{array}$ & $\begin{array}{l}.801 \\
* *\end{array}$ & $\begin{array}{l}.421 \\
*\end{array}$ & $\begin{array}{l}-.850 \\
* *\end{array}$ & & & & \\
\hline Corm weight & $\begin{array}{l}-.727 \\
* *\end{array}$ & $\begin{array}{l}.596 \\
* *\end{array}$ & $\begin{array}{l}.640 \\
* *\end{array}$ & $\begin{array}{l}-.815 \\
* *\end{array}$ & $\begin{array}{l}.738 \\
* *\end{array}$ & $\begin{array}{l}.694 \\
* *\end{array}$ & $\begin{array}{l}.471 \\
* *\end{array}$ & $\begin{array}{l}.660 \\
* *\end{array}$ & $\begin{array}{l}.850 \\
* *\end{array}$ & $\begin{array}{l}.533 \\
* *\end{array}$ & $\begin{array}{l}-.788 \\
* *\end{array}$ & $\begin{array}{l}.893 \\
* *\end{array}$ & & & \\
\hline $\begin{array}{l}\text { Comels per } \\
\text { plant }\end{array}$ & 0.021 & $\begin{array}{l}.389 \\
*\end{array}$ & 0.255 & -0.037 & -0.038 & 0.214 & 0.094 & 0.326 & 0.27 & 0.113 & 0.027 & 0.057 & 0.249 & & \\
\hline $\begin{array}{l}\text { Total cormel } \\
\text { weight }\end{array}$ & -0.232 & 0.338 & -0.021 & -0.204 & 0.12 & 0.299 & -0.003 & 0.135 & 0.081 & 0.098 & -0.072 & 0.271 & 0.239 & $\begin{array}{l}.486 \\
* *\end{array}$ & \\
\hline $\begin{array}{l}\text { Average } \\
\text { Cormel wt }\end{array}$ & -0.2 & -0.133 & -0.251 & -0.088 & 0.018 & -0.146 & -0.125 & -0.259 & -0.237 & -0.239 & -0.143 & 0.198 & 0.075 & $\begin{array}{l}-.449 \\
*\end{array}$ & 0.187 \\
\hline
\end{tabular}

Note. *: Correlation is significant at the 0.05 level; **: Correlation is significant at the 0.01 level; Ht: height; dia: diameter; wt: weight; Veg: vegetative.

\section{Discussion}

Soaking of corms in different solutions of PGRs influenced the sprouting of buds and, also increased the vegetative and total plant height. Usually only one bud sprouted from a corm of gladiolus, but application of benzyl adenine lead to sprouting of multiple sprouts from a single corm in the present research. Benzyl adenine is an important cytokinin and has the capability of multiple shoot induction in several plants and also known as branching agent. The results also confirm the findings of Carey (2008) that exogenous application of benzyladenine promotes multiple shoots in several ornamental plants, including Petunia, Sempervivum, and Salvia. Furthermore, Wróblewska and Dębicz (2013) also reported the increase in the number of lateral buds through the application of benzyladenine in Portulaca umbraticola plants.

The gibberellic acid and ethrel increased the plant height in relatively lower doses and showed reverse effect in their highest concentrations used in this study. The involvement of gibberellic acid in stem elongation process is reported by Sun and Gubler (2004) which are in line with our findings in gladiolus plants. This growth simulative effect of ethrel on plant height and corm production has also been observed by Vijay et al. (2006) in 
gladiolus and this feature has also been observed in Amsterdam, a cultivar of gladiolus, in the present study.

Pre-plant soaking of corms in PGRs showed alterations in the floral characteristics of gladiolus spikes. The gibberellic acid caused the early flowering while bezyl adenine application delayed the flowering. Cytokinins have been reported for induction of early flowering in plants, including Selenicereus and Hylocereus (Khaimov \& Mizrahi, 2006), Gladiolus (Sajjad et al., 2014) which opposes the results of the present study, but there are certain findings which support our results that cytokinin can delay flowering in some plants (Carey, 2008). The possible reason to oppose the present results is the difference in application methods, concentration and stage of growth as Sajjad et al. (2014) used a foliar application method which caused early flowering in gladiolus. As effects of plant growth regulators altered with the change in stages of plant growth on which they are applied (Bakhsh et al., 2011). Gibberellic acid increased the flowering percentage in the present study and these results strengthen by the research work of Gayakvad et al. (2014) that gibberellic acid increased the flowering in Jatropha plants. Ethrel at lower concentration used in this study reduced the flowering time while higher concentration caused delay in flowering. The undesired effect of high concentration of ethrel on flowering of impatiens plants is also reported by Tamari et al. (1998).

The application of benzyladenine increased the number of corms which resulted to raise the corm production twice than the untreated plants. The positive effect of cytokinins to increase the yield of underground structures has been reported on Allium (Pogroszewska et al., 2007), Muscari (Puchalski et al., 1979), Red candyman, a cultivar of gladiolus (Aier et al., 2015) which also support our findings in Amsterdam cultivar of gladiolus. The soaking of corms in ethrel increased the production of corms compared to control plants and the results are in line with the findings of Roychowdhury (1989) that application of ethrel enhances the corm production in gladiolus, and also facilitate the bulb production in tuberose (Nagaraja et al., 1999).

Pre-plant soaking of corms in solutions of PGRs altered the interested variables in gladiolus plants. Application of benzyl adenine induced the multiple shoots and also increased the yield of corms which actually enhanced the multiplication rate of corms more than twice, compared to non treated corms. The research work also indicates that benzyladenine caused delay in flowering while gibberellic acid induced early flowering. The present research work indicated the interesting correlation among vegetative, floral and corm variables. Number of sprouted buds per corm was the most dominant trait found in this study which showed significant influence on the most of valuable traits, including the number of corms produced per plant, which encouraged the rapid multiplication of corms compared to conventional production system.

\section{Acknowledgements}

Author is greatly thankful to Higher Education Commission (HEC) of Pakistan for financial support to carry out the present research work through indigenous scholarship program.

\section{References}

Aier, S., Langthasa, S., Hazarika, D. N., Gautam, B. P., \& Goswami, R. K. (2015). Influence of $\mathrm{GA}_{3}$ and BA on Morphological, Phenological and yield attributes in gladiolus cv. Red Candyman. IOSR Journal of Agriculture and Veterinary Science, 8, 37-42. http://dx.doi.org/10.9790/2380-08623742

Bakhsh, I., Awan, I., Sadiq, M., Niamatullah, M., Zaman, K. U., \& Aftab, M. (2011). Effect of plant growth regulator application at different growth stages on the economical yield potential of coarse rice (Oryza sativa L.). Journal of Animal and Plant Sciences, 21, 612-616.

Boyle, T. H. (1992). Modification of plant architecture in 'Crimson Giant' Easter cactus with benzyladenine. Journal of the American Society for Horticultural Science, 117, 584-589.

Carey, D. J. (2008). The Effects of Benzyladenine on Ornamental Crops (Doctoral dissertation). NCSU Institutional Repository, North Carolina State University, USA. Retrieved from http://repository.lib.ncsu.edu/ir/handle/1840.16/1048

De Munk, W. J., \& Gijzenberg, J. (1977). Flower bud blasting in tulip plants mediated by the hormonal status of the plant. Scientia Horticulturae, 7, 255-268. http://dx.doi.org/10.1016/0304-4238(77)90022-X

Dole, J. M., \& Wilkins, H. F. (1999). Plant growth regulation. Floriculture principles and species (pp. 90-104). Prentice-Hall, Upper Saddle River, N.J.

Gayakvad, P., Jadeja, D. B., \& Bhalawe, S. (2014). Effect of foliar application of $\mathrm{GA}_{3}$, ethrel and copper sulphate on flowering behaviour and sex ratio of Jatropha curcas L. Journal of Applied and Natural Science, 6, 286-289.

Ghamsari, L., Keyhan, E., \& Golkhoo, S. (2007). Kinetics properties of guaiacol peroxidase activity in Crocus 
sativus L. corm during rooting. Iranian Biomedical Journal, 11, 137-146.

Goldblatt, P., \& Manning, J. C. (1998). Gladiolus in Southern Africa: Systematics, Biology, and Evolution (pp. 464). Fernwood Press, Cape Town.

Irish, V. (2009). The flowering of Arabidopsis flower development. The Plant Journal, 61, 1014-1028. http://dx.doi.org/10.1111/j.1365-313X.2009.04065.x

Khaimov, A., \& Mizrahi, Y. (2006). Effects of day-length, radiation, flower thinning and growth regulators on flowering of the vine cacti Hylocereus undatus and Selenicereus megalanthus. The Journal of Horticultural Science and Biotechnology, 81, 465-470.

Larson, R. A., Throne, C. B., Milks, R. R., Iseberg, Y. M., \& Brisson, L. O. (1987). Use of ancymidol bulb dips to control stem elongation of easter lilies grown in a pine bark medium. Journal of the American Society for Horticultural Science, 112, 773-777.

Malabug, L. U., Pompe, C. S. C., Nino, P. M. C. B., Edna, A. A., \& Jose, E. H. (2010). Improving the grain filling and yield of Indica rice through Kinetin (N6-furfuryl adenine) application at flowering stage. The Philippine Journal of Crop Science, 35, 22-35.

Manning, J. C., \& Goldblatt, P. (2008). The Iris Family: Natural History and Classification (pp. 138-142). Timber Press Inco., Portland, Oregon.

Nagaraja, G. S., Gowda, J. V. N., \& Farooqui, A. A. (1999). Effect of growth regulators on growth and flowering of tuberose (Polianthes tubersoa L.) cv. Single. Kamataka Journal of Agricultural Sciences, 12, $236-238$.

Naor, V., Kigel, J., \& Ziv, M. (2005). The effect of gibberellin and cytokinin on floral development in Zantedeschia spp. in vivo and in vitro. Acta Horticulturae, 673, 255-263.

Olszewski, N., Sun, T. P., \& Gubler, F. (2002). Gibberellin signaling: Biosynthesis, catabolism, and response pathways. The Plant Cell, 14, 61-80. http://dx.doi.org/10.1105/tpc.010476

Paulin, A., \& Muloway, K. (1979). Perspective in the use of growth regulators to increase the cut flowers vase life. Acta Horticulturae, 91, 135-141.

Pogroszewska, E., Laskowska, H., \& Durlak, W. (2007). The effect of gibberellic acid and benzyladenine on the yield of (Allium karataviense regel.) 'ivory queen'. Acta Scientiarum Polonorum-Hortorum Cultus, 6, 15-19.

Puchalski, J., Puchalska, M., \& Saniewski, M. (1979). Glucose-6-phosphate dehydrogenase electrophoretic forms during differentiation of benzyladenine induced bulblets of Muscari. Acta Horticulturae, 91, 241-245.

Rademacher, W. (1991). Inhibitors of gibberellin biosynthesis: Applications in agriculture and horticulture. In N. Takahashi, B. O. Phinney \& J. MacMillan (Eds.), Gibberellins (pp. 296-310). Springer-Verlag, New York, N.Y. http://dx.doi.org/10.1007/978-1-4612-3002-1_29

Ranwala, A. P., \& Milller, W. B. (1998). Gibberellin4+7, benzyladenine, and supplemental light improve postharvest leaf and flower quality of cold-stored 'Stargazer' hybrid lilies. Journal of the American Society for Horticultural Science, 123, 563-568.

Ranwala, A. P., Legnani, G., Reitmeier, M., Stewart, B. B., \& Miller, W. B. (2002). Efficacy of plant growth retardants as preplant bulb dips for height control in LA and oriental hybrid lilies. HortTechnology, 12, 426-431.

Roychowdhury, N. (1989). Effect of plant spacing and growth regulators on growth and flower yield of gladiolus grown under polythene tunnel. Acta Horticulture, 246, 259-264.

Sajjad, Y., Jaskani, M. J., Ashraf, M. Y., Qasim, M., \& Ahmad, R. (2014). Response of morphological and physiological growth attributes to foliar application of plant growth regulators in gladiolus "White Prosperity". Pakistan Journal of Agricultural Sciences, 51, 123-129.

Sakai, K., Katano, Y., \& Higuchi, H. (1979). Promoting flowering in Cyclamen persicum by benzyladenine. Research Bulletin of the Aichi-Ken Agricultural Research Center, 11, 81-87.

Schnelle, R., Cerveny, C., \& Barrett, J. (2005). Factors affecting PGR liner dips. Greenhouse Product News, 15, 106-107.

Sun, T. P., \& Gubler, F. (2004). Molecular mechanism of gibberellin signaling in plants. Annual Review of Plant Biology, 55, 197-223. http://dx.doi.org/10.1146/annurev.arplant.55.031903.141753 
Tamari, G., Pappa, L., Zered, T., \& Borochov, A. (1998). Effects of ethrel and gibberellin on impatiens plants. Scientia Horticulturae, 76, 29-35. http://dx.doi.org/10.1016/S0304-4238(98)00128-9

Vijay, K. U., Singh, R. P., \& Singh, A. R. (2006). Influence of ethrel and sand on growth and corm yield of gladiolus cv. congo song. Progressive Agriculture, 6, 143-145.

Wróblewska, K., \& Dębicz, R. (2013). Influnce of time of benzyladenine application on rooting of cuttings and subsequent development of portulaca umbraticola kunth. Acta Scientiarum Polonorum-Hortorum Cultus, $12,89-99$.

\section{Copyrights}

Copyright for this article is retained by the author(s), with first publication rights granted to the journal.

This is an open-access article distributed under the terms and conditions of the Creative Commons Attribution license (http://creativecommons.org/licenses/by/3.0/). 